\title{
ALTERACIÓN HIDROMORFOLÓGICA Y ESTADO ECOLÓGICO DEL TRAMO BAJO DEL RÍO BEMBÉZAR. CUENCA DEL GUADALQUIVIR, ESPAÑA
}

\author{
Belén García-Martínez \\ Universidad de Sevilla \\ mbelen@us.es \\ ORCID iD: https://orcid.org/0000-0002-4589-9385
}

Recibido: 31/07/2020; Aceptado: 04/07/2021; Publicado: 03/12/2021

Cómo citar este artículo/citation: García-Martínez, Belén (2021). Alteración hidromorfológica y estado ecológico del tramo bajo del río Bembézar. Cuenca del Guadalquivir, España. Estudios Geográficos, 82 (291), e071. https://doi.org/10.3989/estgeogr.202082.082

RESUMEN: El río Bembézar, con una cuenca próxima a $2000 \mathrm{~km}^{2}$ de superficie y una capacidad de agua embalsada de $355.5 \mathrm{hm}{ }^{3}$, presenta evidencias de alteración hidromorfológica en su tramo bajo. A partir del estudio de series de caudales (2000-2019) aportadas por el Sistema Automático de Información Hidrológica del Guadalquivir y del análisis de las fotografías aéreas de distintos años (1956 y 2010), las principales alteraciones detectadas son las siguientes: disminución en un $58 \%$ del caudal circulante por el cauce, incremento de la irregularidad estacional con siete meses de aguas bajas, ausencia de caudal ecológico el $66 \%$ de los días, funcionalidad de la llanura de inundación sólo en crecidas extraordinarias, cambio en el patrón morfológico del río, reducción de la anchura media del cauce en un 53\%, incisión generalizada en todo el tramo e invasión de especies exóticas.

Por su parte, la valoración del estado ecológico que realiza de esta masa de agua el Organismo de cuenca competente evoluciona de malo en 2015 a peor que bueno en 2018, como consecuencia de los malos resultados que aportan los indicadores biológicos; pese a que el objetivo medio ambiental para esta masa de agua era el de buen potencial ecológico en 2015 . Se pone de manifiesto, asimismo, la poca idoneidad tanto de los criterios hidromorfológicos utilizados en la definición del estado ecológico como de las actuaciones que se ejecutan para garantizar la conservación de cauces y riberas.

PALABRAS CLAVES: intervención antrópica, régimen fluvial, cambios hidromorfológicos, ecología fluvial, calidad funcional, DMA.

\section{HYDROMORPHOLOGICAL ALTERATION AND ECOLOGICAL STATE OF THE LOWER BEMBÉZAR RIVER. GUADALQUIVIR BASIN, SPAIN.}

ABSTRACT: The Bembézar river, with a basin close to $2000 \mathrm{~km} 2$ of surface and a capacity of impounded water of 355.5 hm3, presents evidence of hydromorphological alteration in its lower section. Based on the study of flow series (2000-2019) provided by the Guadalquivir Automatic Hydrological Information System and the analysis of aerial photographs from different years (1956 and 2010), the main alterations detected in its lower section are the following: decrease by $58 \%$ of the circulating flow through the channel, increase in seasonal irregularity with seven months of low water, absence of ecological flow on $66 \%$ of the days, functionality of the flood plain only in extraordinary floods, change in the morphological pattern of the river, reduction of the average width of the channel by $53 \%$, general incision throughout the section and invasion of exotic species.

The assessment of the ecological status of this waterbody carried out by the competent Basin Authority, evolves from bad in 2015 to worse than good in 2018 , as a consequence of the poor results provided by the biological indicators. The environmental objective for this waterbody was that of good ecological potential in 2015. It also shows the poor suitability of both the hydromorphological indicators used in the definition of the ecological status and the actions that are carried out to guarantee the conservation of riverbeds and banks.

KEY WORDS: anthropic intervention, fluvial regime, hydromorphological changes, river ecology, functional quality, WFD. 


\section{INTRODUCCIÓN}

La respuesta de un río a las modificaciones que experimenta su entorno, ya sean de tipo climático, hidrológico o antrópico, queda registrada tanto a nivel de morfología de su cauce como de su llanura aluvial. Así ha quedado confirmado en numerosos ríos donde las investigaciones destacan importantes ajustes en el comportamiento fluvial tras la construcción de presas (Petts, 1984; Brandt, 2000).

A nivel hidrológico, la magnitud de estos cambios se asocia al grado de regulación del sistema (número de embalses, capacidad y uso del agua), siendo ostensible, entre otros: la reducción genérica del volumen de caudal medio circulante y la alteración del comportamiento estacional (Vanney, 1970; Lorenzo Lacruz et al., 2012; García Martínez, 2016); la pérdida de homogeneidad en el comportamiento hidrológico (García Martínez, 2016); la modificación del transporte sedimentario y de la ecología fluvial (Véricat y Batalla, 2004; García Martínez et al., 2009); así como la reducción de los caudales máximos en avenidas ordinarias (Williams y Wolman, 1984).

Los principales ajustes de dicha alteración hidrológica, a nivel de morfología de cauce, suelen ser los de incisión y confinamiento en un solo cauce (Park, 1977). Esto trae consigo problemas de inestabilidad por socavamiento (Stanley, 1972) en obras de infraestructura, como puentes o carreteras próximas. Otras consecuencias son la divagación lateral y el aumento de la sinuosidad (Thornes, 1977) o la estabilización de antiguos sedimentos (Martínez Castroviejo et al., 1991).

A nivel ecosistémico, es precisamente el régimen de caudales y sus efectos sobre los cauces fluviales el garante de la composición y distribución de la vegetación riparia, así como de su estructura y abundancia (Auble et al., 1994; Auble et al., 2005). Ello repercutirá en la biodiversidad natural y la integridad de los ecosistemas, por lo que el éxito en la conservación de la calidad ecológica y funcional de nuestros ríos, depende de la capacidad de proteger o restaurar los principales aspectos del régimen natural de caudales (Bunn y Arthington, 2002; Ollero et al., 2008).

En este sentido se orienta la Directiva Marco de Agua (2000/60/CE) al pretender armonizar la gestión del recurso con la protección y conservación tanto de las masas de agua como de sus ecosistemas asociados. Para ello, los indicadores hidromorfológicos (DMA, 2000/60/CE) son clave para determinar el estado ecológico de los ríos, en la medida en la que estos procesos son la base fundamental del correcto funcionamiento de los cursos fluviales como ecosistemas. Sin embargo, las importantes afecciones en la dinámica fluvial de muchas acciones humanas siguen sin ser valoradas (Ollero, 2008), tampoco en la definición de la calidad ecológica de muchos ríos.

El río Bembézar, afluente por la derecha del río Guadalquivir al final de su tramo medio, desarrolla una cuenca de drenaje localizada entre las estribaciones de Sierra Morena y la Depresión del Guadalquivir, a unos $50 \mathrm{~km}$ al Oeste de Córdoba (Fig. 1). Su cuenca, próxima a $2000 \mathrm{~km}^{2}$ de superficie, se caracteriza por una precipitación media anual de $700 \mathrm{~mm}$ y un desnivel topográfico general de algo más de $520 \mathrm{~m}$, con una red incidida sobre la topografía general de la Sierra. Con una longitud de algo más de $119 \mathrm{~km}$ y una pendiente media de 0.0043 configura un valle de dirección predominantemente hercínica (NW-SE) hasta Hornachuelos, para tomar dirección N-S al entrar en la cuenca neógena del Guadalquivir.

Este valle queda fragmentado por la presencia de tres embalses, el del Cabril con una capacidad de $0.031 \mathrm{hm}^{3}$ situado en el tramo alto del río, el del Bembézar con $342 \mathrm{hm}^{3}$ de capacidad, en el tramo medio y por último el de Hornachuelos, con una capacidad de $12 \mathrm{hm}^{3}$, en el tramo final de la cuenca, a escasos $10 \mathrm{~km}$ de la confluencia con el Guadalquivir. A ellos se le unen el embalse de Azuaga con $0.54 \mathrm{hm}^{3}$ y el de Guaditoca con $0.95 \mathrm{hm}^{3}$, situados en la cuenca alta del Bembézar, concretamente en los arroyo Sotillos y Guaditoca, respectivamente, ambos afluentes por la derecha del río Bembézar.

El objetivo de este trabajo es aportar algunas claves sobre la alteración que el sistema de embalses Bembézar-Hornachuelos infiere al comportamiento hidromorfológico del río en su tramo bajo. De forma complementaria, se aborda la definición del estado ecológico y el seguimiento que lleva a cabo el Organismo de cuenca en esta masa de agua.

\section{METOdología Y FUENTES}

El análisis hidrológico se realiza a partir de los datos de caudal del río Bembézar facilitados por el Sistema Automático de Información Hidrológica (SAIH) del Guadalquivir (2000-2019) para los embalses del Bembézar (E37) y de Hornachuelos (E38), pese a considerarse datos no contrastados. En ambos casos, el sistema aporta datos tanto de aportación al embalse como de desembalse, diferenciándose, en el caso de Hornachuelos, el desembalse que se realiza directamente al río del que 
FIGURA 1.

LOCALIZACIÓN DE LA CUENCA DEL RÍO BEMBÉZAR

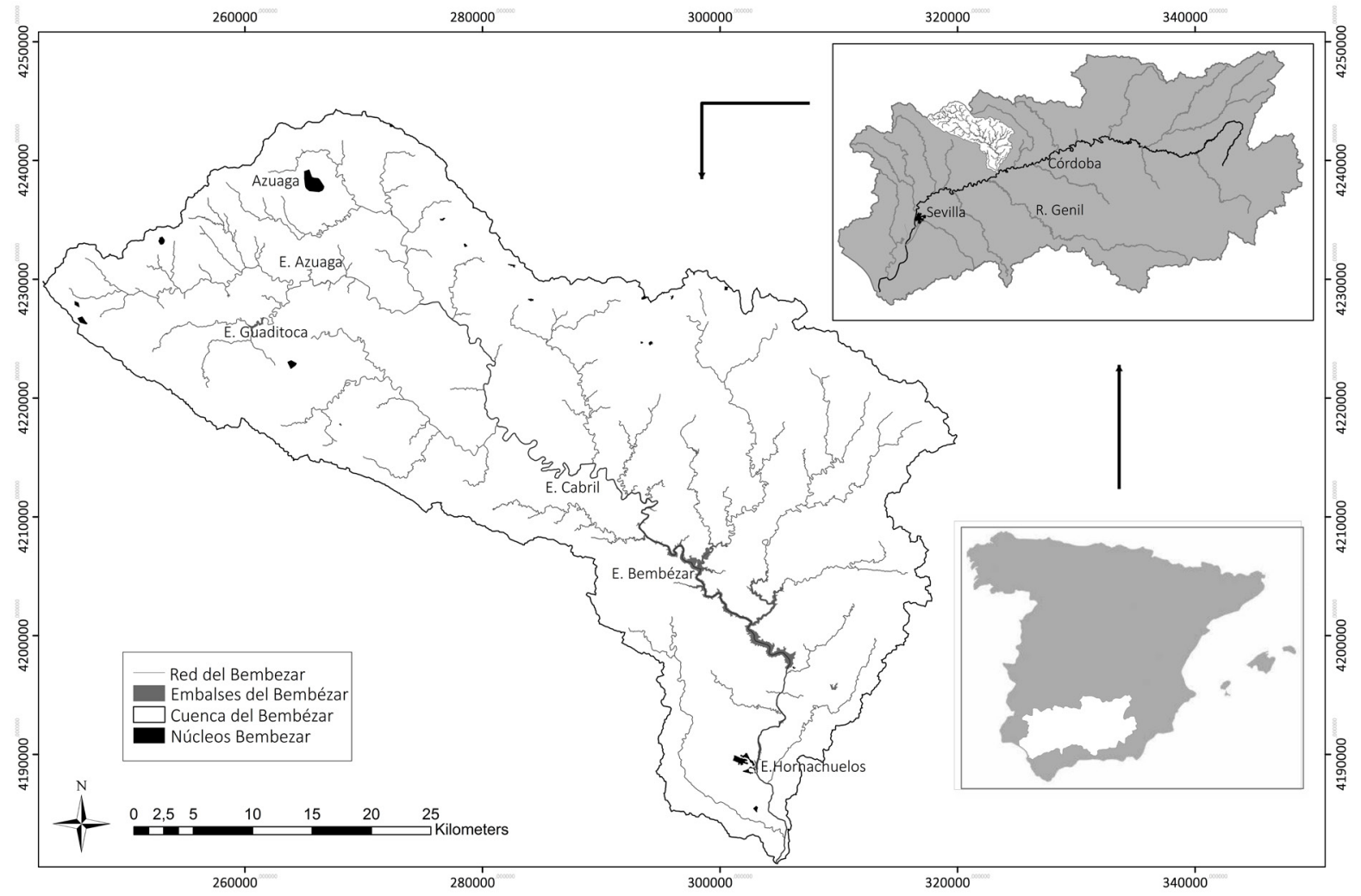

Fuente: Elaboración propia

se destina para el riego. Este hecho permitirá una interpretación más acorde del comportamiento hidrológico del río, sobre todo en lo que respecta a su régimen fluvial y, en consecuencia, a los procesos hidrogeomorfológicos y ecosistémicos que de él puedan derivarse. Se desestiman, por tanto, los datos aportados por el Sistema de Información Anual de Aforos (SIAA) para la cuenca del Bembézar, por no disponer de caudales diferenciados al río en el embalse de Hornachuelos.

La ubicación de ambos embalses en el tramo medio del río permitirá analizar el grado de influencia que la gestión infiere en el comportamiento natural del río Bembézar. Así, aguas arriba del embalse del Bembézar, el comportamiento hidrológico registrado por el río obedece a parámetros naturales para una cuenca de drenaje de $1655 \mathrm{~km}^{2}$ (el $83 \%$ de la cuenca total); si tenemos en cuenta que los embalses de Azuaga y Cabril no alcanzan el 0.6 hm $^{3}$ de capacidad y no ejercen regulación alguna (García Martínez y Ruíz Miranda, 2017). Mientras, aguas abajo de Hornachuelos, y hasta su confluencia con el Guadalquivir, el comportamiento hidrológico del río Bembézar dependerá de la gestión del sistema de embalses.
Para el análisis estadístico de las series de caudal se utiliza el programa AnClim versión 5.025 (Stepánek, 2003) que, junto a la descripción de las series, evalúa la normalidad y homogeneidad de éstas mediante las pruebas de Chi Cuadrado o de Kolmogorov-Smirnov, y Von Neuman, Mann-Kendall o Sperman, respectivamente. Se identifican en cada caso la bondad de ajuste para distintos niveles de significación. En este caso, y aunque algunos autores desconfíen de cualquier aproximación que implique asunciones de normalidad de la serie mediante test de carácter no paramétricos (Aguilar et al., 2002 cfr. Sneyers, 2002), no se utilizarán técnicas absolutas de homogeneización al no contar con una serie lo suficientemente larga que incluya condiciones hidrológicas pre y post embalse, y en la que poder identificar cambios estadísticos en las series.

Teniendo esto último en consideración, y atendiendo a que el caudal modular se ajusta a una distribución normal en condiciones naturales, los posibles cambios en la distribución de los datos del caudal modular pueden ser también un indicador de que el comportamiento hidrológico de un determinado río no obedezca a condiciones estrictamente naturales 
(García Martínez, 2016). En este caso, la variabilidad interanual se interpreta a partir de la representación de los valores estandarizados de la serie, aportando bastante información del comportamiento anual del caudal en condiciones naturales (tramo alto) y derivada de la gestión del sistema de embalses (tramo bajo). Este análisis temporal del caudal, junto con otros aspectos, puede ser clave en la interpretación, no sólo de la evolución hidrogeomorfológica de cauces (reducción de anchura, incisión, acumulación, etc.) y llanuras de inundación (funcionalidad hidrológica) para escalas temporales de decenas de años (Baena, 1998; García Martínez, 2016), sino también como respuesta a agentes antrópicos (Ollero et al., 2004; García Martínez et al., 2009; García Martínez, 2016; García Martínez y Ruiz Miranda, 2017).

El régimen fluvial, como modelo teórico de comportamiento hidrológico, muestra tanto el tipo de alimentación del caudal como la variabilidad estacional respecto al caudal modular de la serie (Remenieras, 1971). Esta variabilidad genera fuertes implicaciones geomorfológicas y ecosistémicas especialmente evidentes a nivel de morfología del cauce (Schumm, 1977) y sus riberas en años hidrológicamente diferentes (Martínez y Fernández Yuste, 2006 cfr. Arthington, 1997). Si tenemos en cuenta que la crecida es el fenómeno hidrológico con mayor capacidad morfogenética sobre el cauce y la llanura, que asegura los ciclos vitales de la vegetación riparia y, por tanto, la conectividad lateral del sistema (Bayley, 1991), el estudio de su variabilidad natural o posible laminación aportará claves para la definición de la calidad ecológica del río.

Por otro lado, los cambios en el patrón morfológico del río han sido definidos mediante la fotointerpretación del vuelo americano de 1956 (situación pre-embalse) y del vuelo de PNOA del año 2010 (situación post-embalse). A partir de la base cartográfica digital existente para cada momento, aportada por el servicio WMS de la REDIAM (Consejería de Medio Ambiente y Ordenación del Territorio, Junta de Andalucía) se realizan sendos esquemas geomorfológicos (programa ArcGis, 10.4.1) en el que quedan representados, en cada caso, el límite de la llanura de inundación natural y/o actual, cauces abandonados, canales de crecida, cauce ordinario, barras laterales y barras de canal.

\section{RESULTADOS}

\section{Regulación antrópica y alteraciones hidrológicas}

La presa del Bembézar (1963) cierra un embalse de $342 \mathrm{hm}^{3}$ de capacidad situado en un contexto pro- ductivo exclusivamente forestal, lo que garantiza unos elevados niveles de calidad del agua que se orienta al abastecimiento, la producción hidroeléctrica y el riego de hasta 15300 ha en el valle del Guadalquivir (CHG, 2002). Aguas abajo, se ubica otro de menor tamaño denominado presa de derivación del Bembézar (1961), conocido también como embalse de Hornachuelos, en el que el nivel del agua se mantiene constante con independencia de la variabilidad de las condiciones climáticas, para llevar a cabo el abastecimiento de los cultivos de regadíos. Desde esta presa parten dos canales, uno por la margen derecha y otro por la izquierda. El canal principal de la margen izquierda tiene una longitud de $11746 \mathrm{~m}$ y un caudal inicial de $4.04 \mathrm{~m}^{3} / \mathrm{s}$, llegando a aportar agua a algo más de 11829 ha de cultivos (CHG, 2002). Por otro lado, el canal de la margen derecha parte desde Hornachuelos y finaliza cerca de la confluencia del arroyo Algarín con el Guadalquivir, totalizando en la actualidad unas 12000 ha de superficie neta (CRMDB, http://bembezar.es/historia/).

\section{Análisis estadístico de los datos}

El análisis estadístico de la serie de caudal de aportación al embalse del Bembézar E37 muestra un módulo de entrada de $6 \mathrm{~m}^{3} / \mathrm{s}$ y una desviación típica de $6.9 \mathrm{~m}^{3} / \mathrm{s}$ para un límite de confianza del $95 \%$. El valor máximo de la serie lo encontramos en el año $2010\left(31.1 \mathrm{~m}^{3} / \mathrm{s}\right)$ considerado también valor extremo y atípico, mientras que el valor mínimo lo tiene el año $2005\left(0.4 \mathrm{~m}^{3} / \mathrm{s}\right)$. Este hecho se concreta en un coeficiente de variación de $115.52 \%$, lo que muestra una gran heterogeneidad en los datos que componen la serie. Por otro lado, la regresión lineal es no significativa, de lo que se infiere la no dependencia o intencionalidad alguna en la regresión. La ausencia de autocorrelación en la serie queda confirmada por el coeficiente de correlación serial (r1) y el test de MannKendall, entre otros (Tabla 1), ratificando por tanto la hipótesis de aleatoriedad de la serie para un nivel de confianza del $95 \%$ (a partir de AnClim, v5.025).

En la serie desembalse del Bembézar E37, se aprecia una disminución leve del módulo del río que supera en poco el $10 \%$ así como de la desviación típica. El valor máximo sigue situándose en el año 2010, aunque con un caudal ligeramente inferior $\left(28,6 \mathrm{~m}^{3} / \mathrm{s}\right)$, manteniéndose la consideración de valor extremo y atípico, mientras que el valor mínimo, ligeramente más elevado $\left(1.3 \mathrm{~m}^{3} / \mathrm{s}\right)$, se desplaza un año, esto es, a 2006. En cuanto a la normalidad o aleatoriedad de la serie, ésta queda confirmada tanto por la regresión lineal no significativa como por los diferentes test no paramétricos utilizados (Tabla 1). 
TABLA 1.

CARACTERÍSTICAS ESTADÍSTICAS DE LAS SERIES DE CAUDALES DEL RÍO BEMBÉZAR (2000-2019)

1A. Serie de Aportación (E37)

\begin{tabular}{|c|c|}
\hline Test & Statist (Confidence level) \\
\hline Kolmogorov-Smirnov test for Normal Distribution & $D=0.277(p=0.094$, O.K.) \\
\hline Tests of Randomness (general) : Serial Correlation Coefficient r1 & r1 = $0.027<$ ? r1 (Tg_95\%) = 0.315 (O.K.) \\
\hline Von Neumann Ratio V & $\mathrm{V}=2.010>$ ? V(Tg_95\%) = 1.371 (O.K.) \\
\hline Test of Randomness (against Trend): Spearman Rank Statistic rs & $\mathrm{rs}=-0.326, \mathrm{t}=-1.465<$ ? Tkrit_97.5\% $=2.100$ (O.K.) \\
\hline Mann-Kendall Rank Statistic & $\mathrm{t}=-0.211<$ ? Tkrit_95\% = 0.318 (O.K.) \\
\hline
\end{tabular}

1B. Serie de Desembalse (E37)

\begin{tabular}{|c|c|}
\hline Test & Statist (Confidence level) \\
\hline Kolmogorov-Smirnov test for Normal Distribution & $D=0.283(p=0.081$, O.K.) \\
\hline Tests of Randomness (general) : Serial Correlation Coefficient r1 & $\mathrm{r} 1=0.020<$ ? r1(Tg_95\%) $=0.315$ (O.K.) \\
\hline Von Neumann Ratio V & $V=2.036>? \mathrm{~V}\left(\mathrm{Tg} \_95 \%\right)=1.371$ (O.K.) \\
\hline Test of Randomness (against Trend): Spearman Rank Statistic rs & $r s=-0.220, t=-0.955<$ ? Tkrit_97.5\% $=2.100$ (O.K.) \\
\hline Mann-Kendall Rank Statistic & $\mathrm{t}=-0.189<$ ? Tkrit_95\% = 0.318 (O.K.) \\
\hline \multicolumn{2}{|l|}{ Linear Regression Model $(x=$ Time $):(y=b 0+b 1 * x): y=6.4183-0.1037^{*} x$} \\
\hline
\end{tabular}

1C. Serie de Desembalse al río (E38)

\begin{tabular}{|c|c|}
\hline Test & Statist (Confidence level) \\
\hline Kolmogorov-Smirnov test for Normal Distribution & $D=0.423(p=0.002$, out $)$ \\
\hline Tests of Randomness (general) : Serial Correlation Coefficient r1 & $\mathrm{r} 1=0.158<$ ? r1(Tg_95\%) = 0.315 (O.K.) \\
\hline Von Neumann Ratio V & $V=1.762>$ ? V(Tg_95\%) = 1.371 (O.K.) \\
\hline Test of Randomness (against Trend): Spearman Rank Statistic rs & $\mathrm{rs}=0.645, \mathrm{t}=3.582<$ ? Tkrit_97.5\% $=2.100$ (out) \\
\hline Mann-Kendall Rank Statistic & $\mathrm{t}=0.242<$ ? Tkrit_95\% $=0.318$ (O.K.) \\
\hline
\end{tabular}

(Source: Like, 1983) AnClim (v5.025)

El comportamiento de la serie desembalse al río de Hornachuelos E38, refleja un descenso del módulo hasta $2.3 \mathrm{~m}^{3} / \mathrm{s}$, siendo la desviación típica similar a la que aportaba la serie de aportación. El valor máximo sigue manteniéndose en el año 2010 (29.1 m³/s), al que se sigue considerando extremo y atípico, junto a los años 2011 y 2013; mientras que el valor mínimo lo comparten los ochos años consecutivos que van desde el 2000 al 2007. Todo ello se traduce en un coeficiente de variación muy elevado, de algo más de $282.46 \%$. Por último, el comportamiento de esta serie reflejaría signos de no aleatoriedad según el test de Kolmogorov-Smirnov para una distribución normal o el test de Spearman, para un límite de confianza del 97.5\% (Tabla 1).

Por tanto, el embalse del Bembézar no ejerce alteración aparente en el comportamiento estadístico de la serie, con excepción del leve descenso del cau- dal modular que circula por el río, y la matización del máximo y el mínimo, éste último también desplazado. Por el contrario, el embalse de Hornachuelos sí altera el comportamiento natural del tramo bajo del río: disminuye drásticamente el caudal modular, en algo más del $58 \%$ respecto al que produce la cuenca alta; incrementa algo más del doble la heterogeneidad en la serie respecto a su comportamiento natural; amplía el número de valores mínimos, de valores extremos y atípicos; y altera, en parte, los signos de normalidad y aleatoriedad del caudal.

\section{LOS EFECTOS DE LA REGULACIÓN EN LA VARIABILIDAD IN- TERANUAL DEL CAUDAL DEL Río BEMBÉzAR}

La variabilidad interanual del comportamiento hidrológico natural (aportacion E37), reflejada en la curva de valores estandarizados (Fig. 2), muestra una alternancia general de años hidrológicamente secos 
FIGURA 2.

VALOR ESTANDARIZADO DEL CAUDAL DEL RÍO BEMBÉZAR (2000-2019)

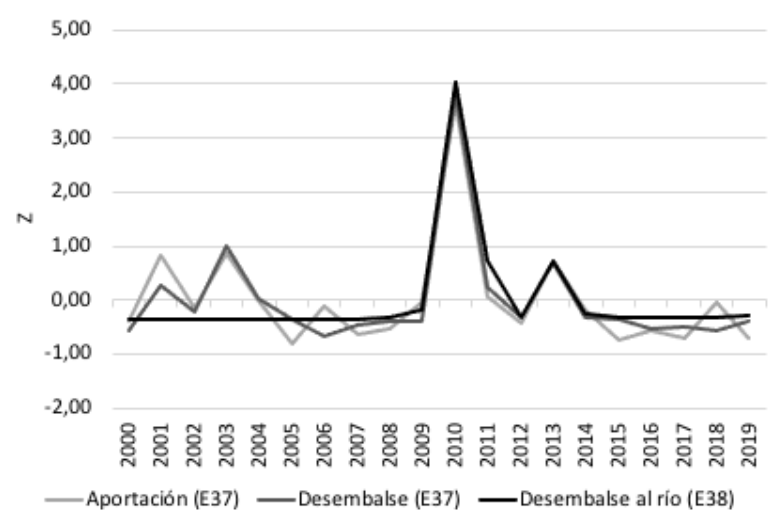

Fuente: Elaboración propia. Datos del SAIH del Guadalquivir

y húmedos, interrumpida por el comportamiento extremo y atípico del año 2010. En el primer decenio se contabilizan dos años muy húmedos (percentil $>80$, en 2001 y 2003) y dos años muy secos (percentil < 20, en 2005 y 2007). En el segundo, se contabiliza un solo año muy humedo (2013) y aumentan los muy secos, que a su vez se hacen más persistentes (2015, 2016, 2017 y 2019). Todo ello infiere un comportamiento irregular de río Bembézar definido por un coeficiente de irregularidad interanual de 77.7.

El comportamiento hidrológico que se desprende del valor estandarizado de los caudales desembalsados por el Bembézar (desembalse E37) mantiene, grosso modo, la misma variabilidad que aguas arriba del embalse (Fig. 2). Sólo se aprecia una cierta regularización del comportamiento en el año 2001 y sobre todo en el periodo comprendido entre 2005-2008 y 2014-2019. Por el contrario, el embalse del Bembézar no suaviza el comportamiento de los años muy húmedos de 2003, 2010 y 2013 (percentil > 90). En términos generales, la gestión del embalse genera una disminución del coeficiente de irregularidad interanual, que en este caso es de 21.7 .

En cambio, el comportamiento hidrológico interanual del río Bembézar, aguas abajo de Hornachuelos (desembalse al río E38), ya no obedece a parámetros naturales (Fig.2). Las características estadísticas descritas en el apartado anterior se manifiestan, a nivel de variabilidad interanual, en una regulación absoluta del embalse durante el periodo $2000-2009$ y 2014-2019, retrayendo al río de funcionalidad alguna durante dichos periodos. Por el contrario, se manifiesta incapaz de hacerlo en años expecialmente húmedos, como es el caso del año 2010 y 2013, ambos definidos como valores atípicos.
Todo ello se traduce en un incremento de la irregularidad interanual hasta un nivel máximo $(\infty)$ en el tramo bajo del río Bembézar. Este comportamiento recuerda al alcanzado en el río Rivera de Huelva, en la cuenca baja del Guadalquivir, con seis embalses y una capacidad de agua embalsada de 460 hm $^{3}$ (García Martínez et al., 2009; García Martínez, 2016).

\section{Cambios en el régimen fluvial del río Bembézar}

El régimen natural del Bembézar (Fig. 3) se caracteriza por un periodo de aguas altas comprendido entre los meses de diciembre a abril y otro de aguas bajas desde mayo a noviembre. En el primero se identifica un máximo principal en diciembre y otro secundario en marzo, en ambos casos superando el coeficiente 2 , y separados por una pequeña inflexión en enero que no desciende el caudal modular. A partir de ese momento el caudal irá descendiendo hasta el mes de junio, cuando se incia el periodo de estiaje que se hace extremo hasta el mes de septiembre, con valores de caudal próximo a cero. El mes de octubre, mes en el que comienza el año hidrológico, evidencia una recuperación de caudal que se mantiene con ligero ascenso en el mes de noviembre, llegando a superar en este caso el módulo de la serie.

FIGURA 3.

CAMBIOS EN EL RÉGIMEN FLUVIAL DEL RÍO BEMBÉZAR (2000-2019)

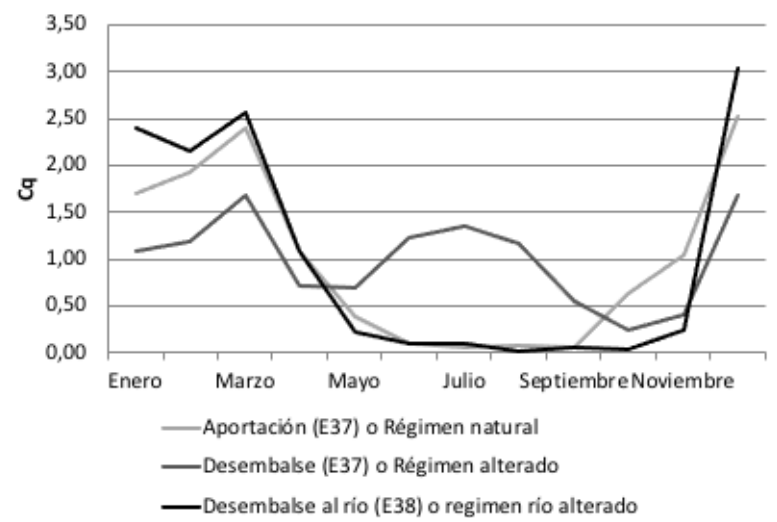

Fuente: Elaboración propia. Datos del SAIH del Guadalquivir

Sobre la base de todo lo anterior, el régimen fluvial del río Bembézar en su cuenca alta quedaría englobado en lo que Masachs (1948) definió como régimen pluvial mediterráneo subtropical, aún cuando se alcancen coeficientes de caudal superior a 2, definido por Masachs (1948) como valor crítico para un pluvial subtropical.

Por el contrario, la configuración del régimen con los datos de desembalse (E37) nada tiene que ver con 
el comportamiento natural que el río adquiere en su cabecera. Se identifican dos periodos de aguas altas, uno que iría desde diciembre a marzo y otro desde junio a agosto. De la misma manera, se identifican dos periodos de aguas bajas, el primero en los meses de abril y mayo, y el segundo entre septiembre y noviembre. Se mantienen los máximos naturales de diciembre y marzo, aunque con coeficientes de caudal inferior (1.8) y se identifica un nuevo máximo, de menor entidad y de origen antrópico, en el mes de julio (1.3). Con ello, desaparece el estiaje de verano que se desplaza, en parte, al mes de octubre.

El régimen que describe el río aguas abajo del embalse de Hornachuelos (E37) vuelve a recuperar los periodos de aguas altas y bajas estacionales, en inviernoinicio de la primavera y en verano, respectivamente, aunque en ambos casos con visibles rasgos de antropización. En este sentido, el periodo de aguas altas se reduce a cuatro meses (de diciembre a abril), y los máximos de diciembre y marzo se extreman, con coeficientes de caudal por encima de 3 y 2,5 respectivamente; trasladándose la típica inflexión subtropical de enero al mes de febrero. Por otro lado, el periodo de aguas bajas se amplía a siete meses (de mayo a noviembre), con estiaje acusado entre los meses de agosto a octubre. En este contexto, el comienzo del año hidrológico no se manifiesta en un incremento del caudal en el cauce, sino que éste queda retenido en el embalse.

Con ello podemos concluir que los primeros síntomas en la alteración del régimen fluvial del río Bembézar están asociados a la gestión del embalse del Bembézar, que invierte por completo el régimen para así poder abastecer de agua a los cultivos de regadío en el periodo estival. Síntomas de alteración detectados también en otros afluentes del bajo Guadalquivir, como es el caso del río Guadalbarcar (García Martínez, 2016). Estos primeros síntomas dan paso a una alteración más extrema en el tramo bajo del río, aguas abajo del embalse de Hornachuelos. En este caso, al extremarse el comportamiento diferenciado entre máximos y mínimos (siete meses sin superar el 0.25 de coeficiente de caudal), el régimen incrementa la irregularidad intranual (> 300). Este comportamiento del río Bembézar en su tramo final se asimila al manifestado en el río Rivera de Huelva (García Martínez y Baena, 2001; García Martínez, 2016).

\section{La regulación del sistema de embalses en las crecidas fluviales del río Bembézar}

De todas las crecidas que tienen lugar en el bajo Guadalquivir en los últimos veinte años (años 2001,
$2003,2010-2011$ y 2013) y que han sido puestas de manifiesto en otros trabajos (García Martínez y Baena, 2002; García Martínez y Baena, 2007; García Martínez, 2016), se estudian las generadas por el río Bembézar en los años 2001 y 2010, así como el efecto regulador que tiene sobre ellas el sistema de embalses Bembézar-Hornachuelos.

La primera crecida natural de la serie tiene un tiempo de base de tres días y es la que acontece en enero 2001 (Fig. 4A), dando comienzo el 10 de enero y alcanzando en un sólo día el valor máximo de caudal o punta de crecida $\left(580.7 \mathrm{~m}^{3} / \mathrm{s}\right)$. Su evacuación natural también muy rápida, tiene lugar en un sólo día, iniciándose la curva de agotamiento el 13 de enero. Sin embargo, y pese a ser un evento rápido y de gran magnitud, ésta no tiene manifestación alguna en el cauce aguas abajo de Hornachuelos, quedando retenida por completo en el embalse y contraemblase del Bembézar.

FIGURA 4

\section{EFECTO REGULADOR DEL SISTEMA DE EMBALSES BEMBÉZAR-HORNACHUELOS EN MÁXIMOS HIDROLÓGICOS}

\section{A. CRECIDA DE ENERO DE 2001}

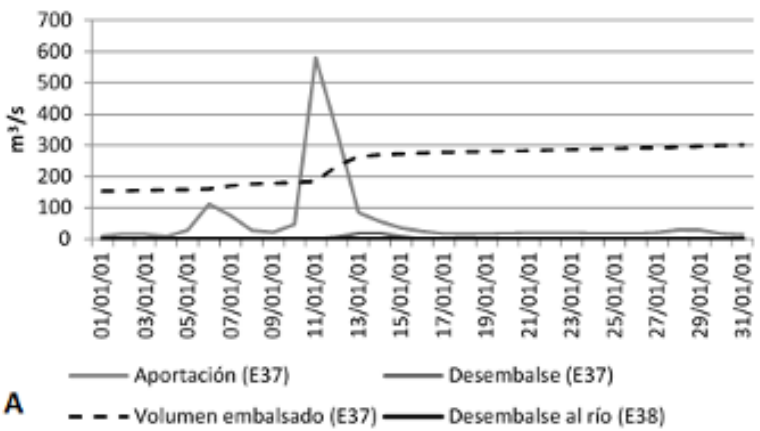

\section{B. CRECIDA DE DICIEMBRE DE 2010-ENERO DE 2011}

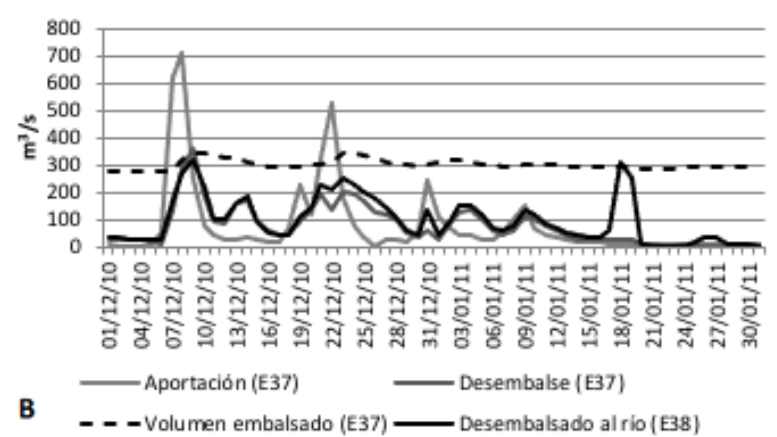

Fuente: Elaboración propia. Datos del SAIH del Guadalquivir

La correspondiente al año 2010 (Fig. 4B) se inserta en un año especialmente húmedo desde un punto de 
vista climático (MMA, 2011) e hidrológico en el bajo Guadalquivir (García Martínez, 2016). En este caso, la crecida natural del Bembézar genera cuatro ondas con puntas de crecida decrecientes, con caudales medios máximos diarios de $708 \mathrm{~m}^{3} / \mathrm{s}$ el 8 de diciembre de 2010 (con valores de caudales máximos de 2697 $\mathrm{m}^{3} / \mathrm{s}$ ), de $528 \mathrm{~m}^{3} / \mathrm{s}$ el 22 de diciembre, de $244 \mathrm{~m}^{3} / \mathrm{s}$ el 31 de diciembre y de $147 \mathrm{~m}^{3} / \mathrm{s}$ el 9 de enero, iniciándose el 10 de enero la curva de agotamiento. Aguas abajo, el embalse del Bembézar en su intento de laminarla, genera otras tantas ondas, aunque con caudales mucho menores que oscilan entre los 362 y los 97 $\mathrm{m}^{3} / \mathrm{s}$. La manifestación de este evento en el río Bembézar, aguas abajo de Hornachuelos, refleja un comportamiento similar al descrito con anterioridad, aun cuando en este caso se evidencia otra onda de crecida más con un caudal medio máximo diario superior a $300 \mathrm{~m}^{3} / \mathrm{s}$. Esta onda de crecida a la que nos referimos se identifica entre los días 17 y 19 de enero, manifestándose en la llanura de inundación del tramo bajo del Bembézar una vez finalizada la crecida natural.

De ello se desprende que el sistema de embalses del río Bembézar tienen capacidad para retener las crecidas ordinarias o las correspondientes a años húmedos, cuando los volúmenes de caudal embalsado previos no superan los $150 \mathrm{hm}^{3}$. En este caso, la llanura aluvial del Bembézar no adquire funcionalidad hidrológica alguna en su tramo bajo. Mientras que en años especialmente húmedos con sucesión de ondas de crecida y volúmenes de agua embalsada previos de en torno a $300 \mathrm{hm}^{3}$, el sistema de embalses Bembézar-Hornachuelos no las retiene. Antes bien, genera ondas de crecida inducidas de carácter antrópico, prolongando durante más tiempo la funcionalidad de la llanura de inundación del tramo bajo del río Bembézar.

\section{IMPLICACIONES ECOSISTÉMICAS AGUAS ABAJO DE LA PRESA DE HORNACHUELOS}

La configuración histórica del trazado del Bembézar en su tramo bajo ha respondido siempre a los cambios de su nivel de base, el Guadalquivir, tal y como queda puesto de manifiesto en la cartografía histórica consultada (Francisco Gozar, 1768; J.A. de Larramendi, 1819 y Mapa Topográfico Nacional, 1918). De esta manera, la dinámica lateral del Guadalquivir iniciada a finales del siglo XVIII culmina con un cambio en la confluencia del Bembézar algo más de 1,4 km al sur, en los primeros decenios del siglo XIX; consolidándose grosso modo en esa posición desde entonces y hasta la actualidad.

Sin embargo, desde la segunda mitad del siglo XX, los principales cambios hidromorfológicos y ecosisté-
FIGURA 5.

CONFIGURACIÓN MORFOLÓGICA NATURAL DEL RÍO BEMBÉZAR EN SU TRAMO BAJO (AÑO 1956)

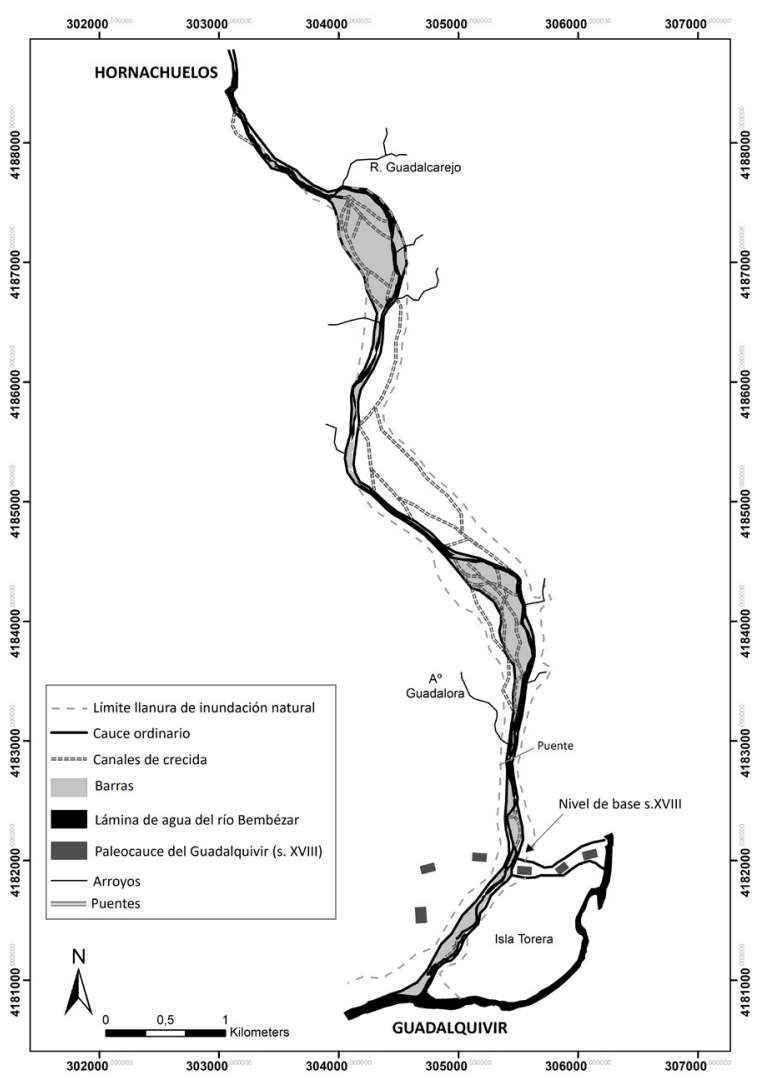

Fuente: Elaboración propia

micos que se detectan en el cauce y su llanura se derivan de la influencia del sistema de presas BembézarHornachuelos. El seguimiento diacrónico del trazado del río Bembezar, en su tramo bajo (Fig. 5 y 6), en condiciones hidromorfológicas distintas, pre-embalses (año 1956) y post-embalses (año 2010) infiere cambios en la calidad del ecosistema fluvial.

Aguas abajo del embalse de Hornachuelos, el río permanece encajado en las calizas del Cámbrico hasta aproximadamente la confluencia del río Guadalcarejo, afluente del Bembézar por su margen izquierda (Fig. 5). A partir de entonces, el río deserrolla una llanura de inundación prácticamente homogénea en anchura (con una anchura máxima superior a $600 \mathrm{~m}$ ) que se extiende hasta la confluencia del arroyo Agualora. En su tramo final, ésta se estrecha hasta un $75 \%$ como consecuencia del encajamiento del río en las terrazas bajas del Guadalquivir.

En este contexto, el trazado del río para mediados del siglo XX queda configurado por un cauce ordinario ancho $( \pm 400 \mathrm{~m}$ ) y poco profundo, con proliferación de 
FIGURA 6.

CAMBIOS HIDROMORFOLÓGICOS EN EL RÍO BEMBÉZAR AGUAS ABAJO DE LA PRESA DE HORNACHUELOS (AÑO 2010)

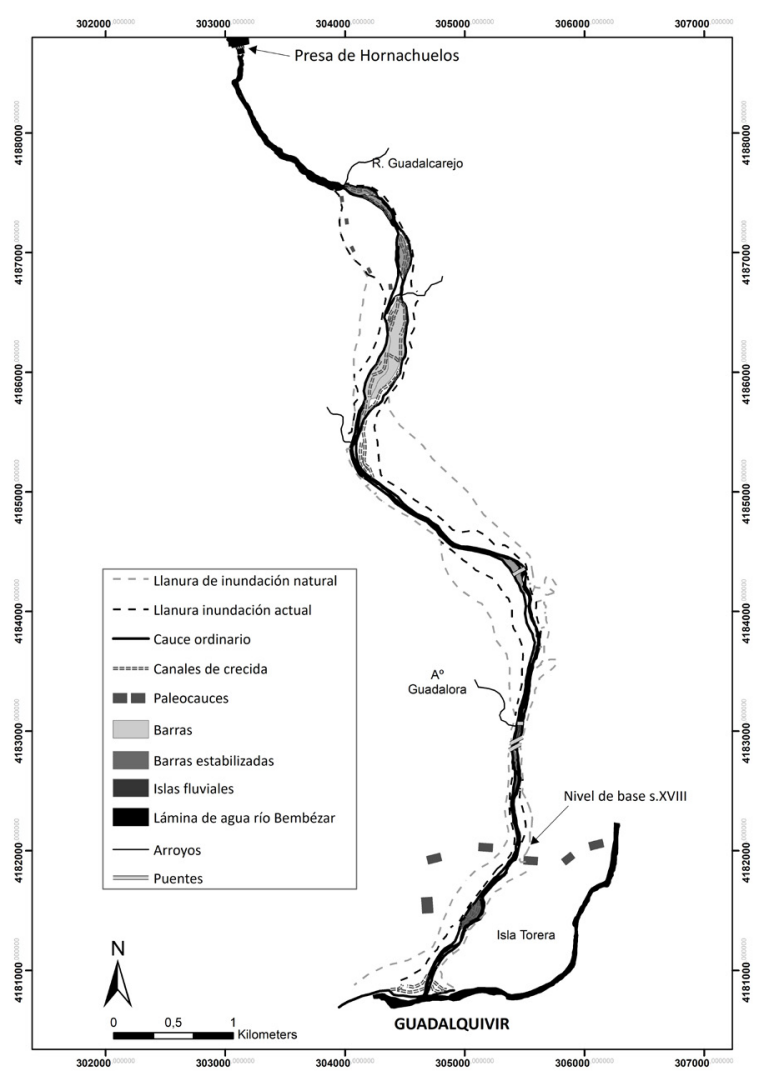

Fuente: Elaboración propia

barras de cauce y canales de crecida, especialmente reconocibles aguas abajo y aguas arriba de las confluencias de los arroyos Guadalcarejo y Agualora respectivamente; describiendo en ambos tramos un patrón morfológico de tipo trenzado. Entre ellos, y pese a ser reconocible en la llanura signos evidentes de este modelo, el río configura un cauce ordinario más estrecho $( \pm 80 \mathrm{~m})$, con menor presencia de barras y algo más sinuoso. Por su parte, el tramo de desembocadura, encajado en la llanura aluvial del Guadalquivir, conforma un cauce ordinario con una anchura media de $100 \mathrm{~m}$ y presencia de barras laterales al cauce.

Con la construcción del sistema de presas BembézarHornachuelos en la década de los sesenta se interrumpe el tránsito sedimentario de carga grosera desde el tramo alto, lo que se traduce en una reducción de la anchura del cauce ordinario (53\%) en el tramo bajo del río Bembézar en 2010 (Fig. 6), con menor presencia de barras centrales y laterales en el cauce (post-embalse). Asimismo, la disminución del volumen de caudal circulante (superior al 58\%), la modificación del comporta- miento estacional a más extremo, con un incremento de la irregularidad superior a 985\%; y las tareas de desembalses de "aguas limpias" en años hidrológicamente húmedos generan incisión en el lecho y acorazamiento aguas abajo de la presa de Hornachuelos. Esto se traduce en un cambio del patrón morfológico del río para comienzos del siglo XXI, caracterizado por un único cauce más estrecho ( $\pm 52 \mathrm{~m}$ de anchura media), sinuoso e incidido en su llanura ( $>3 \mathrm{~m}$ en el puente mirador).

Ello conlleva pérdida de la calidad del ecosistema fluvial (Fig. 7). Por un lado, porque la incisión progre-

FIGURA 7.

IMPLICACIONES DE LA ALTERACIÓN DEL RÉGIMEN FLUVIAL DEL RÍO BEMBÉZAR EN:

A. LA CALIDAD DEL ECOSISTEMA FLUVIAL B. EL MODELO DE PATRÓN FLUVIAL
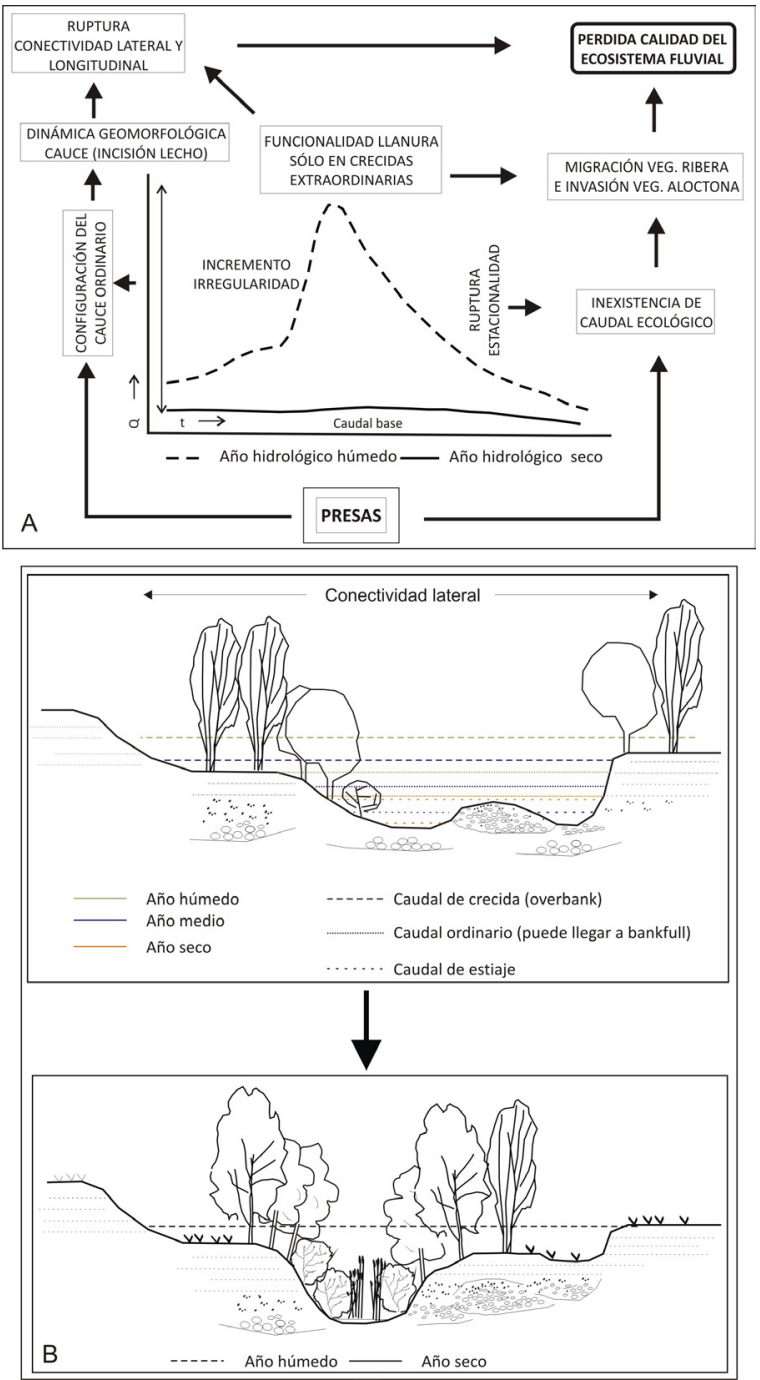

Fuente: A. Basado en Martínez y Fernández Yuste, 2006, cfr. Arthington, 1997; B. Elaboración propia 
siva del lecho junto al control antrópico de las crecidas ordinarias genera una ruptura de la conectividad lateral del cauce del Bembézar con su llanura de inundación; quedando su funcionalidad supeditada al régimen de caudal extraordinario, parcialmente laminado por las presas (Fig. 7A). La pérdida de funcionalidad ordinaria de la llanura de inundación, con el correspondiente descenso del nivel freático, y el incremento y persistencia de los meses de estiaje, ha generado una migración progresiva de la vegetación de ribera hacia el lecho para satisfacer sus necesidades hídricas. Esto lleva consigo la invasión del cauce ordinario y la obstaculización a la laminación de una hipotética crecida por desembalse (Fig. 7B). Este confinamiento de la vegetación de ribera a las márgenes del cauce se ve reforzada, más si cabe, por el avance de los cultivos de regadío que ocupan la totalidad de la llanura, especialmente en la margen derecha del río.

Por otro lado, la contabilización del número de días con un desembalse de $0 \mathrm{~m}^{3} / \mathrm{s}$, en los últimos diez años (2698 días), determina que el río Bembézar no ha presentado funcionalidad hídrica en su tramo bajo el $66 \%$ de los días. En este sentido, la no implementación de un régimen de caudal ecológico, acorde con el régimen natural del río Bembézar, dificulta la conservación de los valores ecológicos propios del cauce y sus riberas en su tramo bajo. En ellas, roto el ciclo vital natural de la vegetación de ribera, empiezan a proliferar especies exóticas como el ailanto (Ailanthus altissima), el eucalipto (Eucalyptus) o la caña común (Arundo donax) que se adaptan mejor a unas condiciones hídricas más extremas, compitiendo así con las especies de ribera. También la eutrofización creciente de las aguas del río, como consecuencia de la escasa torrencialidad natural y la disminución de humedad de los suelos riparios, son clave en la expansión de esta flora invasora. En este sentido, las riberas han ido perdiendo el gradiente natural de su humedad ordinaria y las barras de gravas permeables han sido sustituidas por sedimentos arcillosos, por lo que la flora exótica ha ido creciendo también con mayor facilidad en las riberas.

Por tanto, modificada la funcionalidad del cauce del Bembézar y de su llanura de inundación actual, éste va perdiendo su condición de "río" para pasar a convertirse en un canal de desagüe del caudal en crecida extraordinaria. Mientras que la ausencia de caudal ecológico no sólo supone la degradación de las riberas del Bembézar, sino que trae consigo una merma de las funciones ecosistémicas, ambientales y paisajísticas del río. Asimismo, la intervención hidráulica sobre el río genera un cambio en la configuración del lecho del río Bembézar, que se adapta a las nuevas condiciones morfodinámicas para garantizar de manera óptima la evacuación del caudal ordinario y la carga transportada. Esto supone un cambio en el patrón morfológico fluvial que pasa de un modelo de cauce ancho, poco profundo y diversidad de barras y canales de crecida, a otro de cauce único, más estrecho, incidido y algo más sinuoso.

\section{DisCUSIÓN Y CONCLUSIONES}

El sistema de embalses Bembézar-Hornachuelos trae consigo la alteración hidromorfológica del río Bembézar en su tramo bajo.

Junto al descenso en un $58 \%$ del caudal circulante por el cauce, éste ve incrementada su heterogeneidad adquiriendo protagonismo los comportamientos extremos y atípicos, y apreciándose signos de no aleatoriedad en la variabilidad del caudal. Su variabilidad interanual refuerza el comportamiento funcional del río sólo en años muy húmedos, en los que el sistema de embalses infiere cierta incapacidad de regulación en crecidas extraordinarias. Orientado a satisfacer las necesidades hídricas de las explotaciones de regadío, el embalse del Bembézar invierte el régimen fluvial natural del río, extremándose su estacionalidad aguas abajo del embalse de Hornachuelos, con hasta siete meses de aguas bajas y estiajes acusados, con el $66 \%$ de los días sin desembalses al río $\left(0 \mathrm{~m}^{3} / \mathrm{s}\right)$.

Este modelo de comportamiento se observa grosso modo en otros ríos del ámbito mediterráneo como es el caso de los ríos Ebro, Júcar y Segura entre otros (Frutos et al., 2004; López Bermúdez, 2004; Gil Olcina, 2006). Sin embargo, sobre la pérdida de aleatoriedad en el comportamiento interanual imputable a esta regulación, sólo encontramos las aproximaciones al análisis de la homogeneidad de las series de caudales en el bajo Guadalquivir (García Martínez, 2016), mediante técnicas absolutas de homogeneización consistente en la búsqueda de cambios en los estadísticos de las series analizas. En el caso del Bembézar debemos aludir una vez más, al carácter aproximativo de este tipo de análisis mediante test de carácter no paramétricos, al no contar con una serie lo suficientemente larga que incluya condiciones hidrológicas pre y post embalse.

Esta desnaturalización hidrológica conlleva la alteración del patrón morfológico del tramo bajo del Bembézar, similar al de otros ríos peninsulares (Ollero et al., 2004; Conesa y Pérez, 2014; Baena et al., 2019; 
TABLA 2

EVOLUCIÓN DEL ESTADO ECOLÓGICO DEL TRAMO BAJO DEL RÍO BEMBÉZAR

\begin{tabular}{|c|l|c|c|c|c|c|}
\hline \multicolumn{7}{|c|}{ Primer Ciclo de Planificación (2009-2015) } \\
\hline CODMASA & \multicolumn{1}{|c|}{ Masa de agua } & E.Biológico & E.Morfológico & E. Fis-Quim. & E. Ecológico & Previsión OMA \\
\hline ES0511100094 & $\begin{array}{l}\text { Río Bembézar aguas } \\
\text { abajo de la presa de } \\
\text { Hornachuelos }\end{array}$ & Malo & Bueno & Muy bueno & Malo potencial \\
ecológico en 2015
\end{tabular}

\begin{tabular}{|l|c|c|c|c|c|c|c|}
\hline \multicolumn{9}{|c|}{ Segundo ciclo de planificación (2016-2021) } \\
\hline Masa de agua & $\begin{array}{c}\text { Estado } \\
\text { ecológico } \\
\text { Plan 2015 }\end{array}$ & $\begin{array}{c}\text { Estado ecológico } \\
\text { del seguimiento } \\
2015-2016\end{array}$ & $\begin{array}{c}\text { Estado ecológico } \\
\text { del seguimiento } \\
2016-2017\end{array}$ & $\begin{array}{c}\text { Estado global } \\
\text { del seguimiento } \\
2015-2016\end{array}$ & $\begin{array}{c}\text { Estado global } \\
\text { del seguimiento } \\
2016-2017\end{array}$ & $\begin{array}{c}\text { Estado ecológico } \\
\text { del seguimiento } \\
2017-2018\end{array}$ & $\begin{array}{c}\text { Evolución } \\
\text { ecológico } \\
\text { Plan 2018 }\end{array}$ \\
\hline $\begin{array}{l}\text { Río Bembézar } \\
\text { aguas abajo } \\
\text { de la presa de } \\
\text { Hornachuelos }\end{array}$ & Malo & No valorado & Malo & No valorado & Peor que bueno & Bueno & Mejora \\
Peor que \\
bueno
\end{tabular}

Fuente: Planes Hidrológicos de la Demarcación Hidrográfica del Guadalquivir

Talavera y Sánchez, 2019). El control hidrológico junto con la interrupción del tránsito sedimentario determina el déficit sedimentario en el cauce actual, reajustándose este desequilibrio dinámico con una progresiva tendencia a la incisión, el estrechamiento del cauce y al acorazamiento del lecho aguas abajo del embalse de Hornachuelos. Esto se traduce en la configuración actual de un cauce único más estrecho e incidido respecto a una llanura de inundación que queda colgada, en la actualidad, respecto a la variabilidad del caudal ordinario y a su nivel freático.

Esto supone una ruptura de la conectividad lateral del cauce con su llanura de inundación, que funciona sólo en años hidrológicamente húmedos, cuando el sistema de embalses es incapaz de retener las crecidas extraordinarias. Por tanto, el sistema de presas modifica la funcionalidad del cauce del Bembézar y de su llanura de inundación perdiendo la condición de "río" para transformarse en un canal de desagüe del caudal en crecida extraordinaria.

Por su parte, las riberas, alterada la variabilidad estacional del régimen hidrológico y del caudal ecológico del río, experimentan la merma de sus funciones ecosistémicas, ambientales y paisajísticas, mediante la invasión de especies alóctonas y una migración progresiva hacia el cauce; lo que aporta una mayor rugosidad al lecho y una mayor turbulencia en la evacuación del caudal de crecida.

Pese a ello, la catalogación que se hace del estado morfológico para esta masa de agua, en el primer ciclo hidrológico (2009-2015) es de bueno (Tabla 2), siendo el estado biológico y físico-químico malo y muy bueno, respectivamente (Plan Hidrológico del Guadalquivir, primer ciclo). Como consecuencia, el es- tado ecológico de esta masa de agua fue catalogado como malo, imperando los parámetros biológicos por encima del resto, con la previsión de buen potencial ecológico para 2015.

A este respecto, si bien es cierto que la variable hidrogeomorfológica tiene un menor peso específico en la calificación final del estado ecológico de las masas de agua, no es menos cierto que esta variable es esencial a la hora de poder definir el estado de la misma. Así se puso de manifiesto con la entrada en vigor de la Directiva Marco de Agua (2000/60/CE), ofreciendo una nueva perspectiva para la conservación y ordenación de los sistemas fluviales basada en la necesidad de aplicar las medidas necesarias para prevenir el deterioro del estado de las aguas superficiales, así como proteger, mejorar y regenerar todas las masas de agua.

Sin embargo, el grado de valoración de esta componente hidrogeomorfológica ha sido muy distinto de unas demarcaciones a otras. En el caso de la demarcación del Guadalquivir, los indicadores hidrogeomorfológicos $^{1}$ (Extracción, QBR, IHF, CBRf) se circunscriben casi exclusivamente al estado de la vegetación de ribera, sin que se analicen en ningún caso, parámetros morfohidrológicos de los que se derive la caracterización, alteración y/o calidad hidromorfológica de los cauces y las riberas de manera correcta. Ello explicaría que el tramo bajo del río Bembézar fuese catalogado en el primer ciclo hidrológico (2009-2015) con un estado morfológico bueno. La catalogación de bueno que se le otorga al índice de extracciones indicaría,

1 Extracción: índice de alteración hidrológica QBR: Qualitat del Bosc de Ribera

IHF: Índice de Hábitat Fluvial

CBRf: Calidad del Bosque de Ribera por fotointerpretación 
según esta valoración, una escasa alteración del comportamiento hidrológico del río aguas abajo de la presa de Hornachuelos. Por otro lado, el seguimiento del estado ecológico que se hace de esta masa de agua en el siguiente ciclo de planificación (2015-2020), si bien es cierto que mejora la evolución del seguimiento a lo largo de este ciclo, el estado ecológico del Plan no mejora. Así, lejos de alcanzar la previsión de buen potencial ecológico para 2015, la masa de agua aguas abajo del embalse de Hornachuelos presenta una calificación peor que bueno en 2018.

Entre las propuestas de mejora para la consecución de los objetivos ambientales el plan incluía un proyecto de "Restauración ambiental del tramo bajo del rio Bembézar y su entorno fluvial" (Plan Hidrológico, primer ciclo hidrológico, Anejo 10) incluido en la Estrategia Nacional de restauración de ríos (https:// www.miteco.gob.es/es/agua). Este tipo de actuaciones dirigidas fundamentalmente a la retirada de vegetación muerta, residuos sólidos y eliminación de la vegetación exótica e invasora, asociada por otro lado a la desnaturalización hidrológica provocada por el sistema de embalses; se suma a las ya proyectadas por la Confederación del Guadalquivir en su "Plan de limpieza de cauces y arroyos". Así, lo que en principio pudiera considerarse como una gran inversión en materia de conservación y mantenimiento de cauces, persigue en realidad una mejora de la eficiencia hidráulica mediante el aumento de la sección de desagüe y la reducción de la rugosidad de los cauces para minimizar el problema de inundaciones asociados a desembalses rápidos. Para ello, se modifica la morfología del cauce, se elimina la vegetación viva y el efecto regulador que tiene en el funcionamiento de los ríos y se extrae la madera muerta, empobreciendo ecológicamente las riberas.

Estas prácticas muy cuestionadas, tanto desde la óptica de la dinámica fluvial como desde la aplicación de la normativa europea (Cabrero Rodríguez y Magdaleno Mas, 2015), lejos de mejorar la calidad ecológica del tramo bajo del Bembézar, puede llevar asociados la reactivación de procesos de erosión lateral, incisión en el lecho, inhibición de procesos morfodinámicos, o la destrucción de vegetación de ribera y hábitats, tal y como se desprende de otros trabajos (Ollero, 2013; Herrera Grau, 2014; Cabrero Rodríguez y Magdaleno Mas, 2015); agravando así la alteración hidrogeomorfológica vinculada al sistema de embalses.

Con todo, veinte años después de la entrada en vigor de la DMA se hace más necesario que nunca la incorporación de mecanismos de evaluación hidro- morfológica específicos, claros y contrastables en la definición del estado ecológico de los ríos de la demarcación del Guadalquivir. La aplicación de métodos como el Índice Hidrogeomorfológico (IHG) (Ollero et al., 2008) o el Morphological Quality Index (MQI) y su nueva extensión Hydro-Morphological Quality Index (HMQI) (Rinaldi et al, 2013; Rinaldi et al., 2017) están dando buenos resultados tanto en la cuenca del río Ebro (Ollero et al., 2008) como en el propio Guadalquivir (Rinaldi et al., 2018; Rinaldi et al., 2020). Sin embargo, el Ministerio para la Transición Ecológica parece apostar por el Protocolo de caracterización hidromorfológica (MITECO, 2019), una revisión mejorada del Protocolo de 2015. Su aplicación podría ser de obligado cumplimiento en el tercer ciclo de planificación hidrológica (2021-2027), en el que afloran como temas importantes las alteraciones hidromorfológicas y la implantación del régimen de caudales ecológicos. La aplicación de este Protocolo por los Organismos de cuenca en los próximos años, además de poner de manifiesto la viabilidad del mismo para la definición de la calidad ecológica de los ríos, abriría un nuevo horizonte algo más esperanzador en la gestión fluvial.

\section{AGRADECIMIENTOS}

Este trabajo es una contribución al Grupo de Investigación Geografía Física Aplicada y Patrimonio (HUM697).

\section{BIBLIOGRAFÍA}

Aguilar, E., Brunet, M., Saldié, O. Sigró, J. y López, D. (2002). Hacia una aplicación óptima del Standard Normal Homogeity Test para la homogeneización de series temporales, En La información climática como herramienta de gestión ambiental, bases de datos y tratamientos de series climatológicas : VII Reunión Nacional de Climatología, (pp. 17-33), Albarracín.

Auble, G.T., Friedman, J.M., Scott, M.L. (1994). Relating riparian vegetation to present and future streamsflows. Ecological Applications 4(3), 544554.

Auble, G. T., Scott, M. L., \& Friedman, J. M. (2005). Use of individualistic streamflow-vegetation relations along the Fremont River, Utah, USA to assess impacts of flow alteration on wetland and riparian areas. Wetlands, 25(1), 143-154.

Baena Escudero, R. (1998). Sistemas fluviales mediterráneos: ritmos hidrológicos, inestabilidad de cauces y vertientes, riesgos y nuevas metodolo- 
gías de investigación, En Cuaternario del litoral y entorno continental del mar de Alborán, (pp. 5759), Melilla.

Baena-Escudero, R., Rinaldi, M., García-Martínez, B., Guerrero-Amador, I. C., y Nardi, L. (2019). Channel adjustments in the lower Guadalquivir River (southern Spain) over the last 250 years. Geomorphology, 337, 15-30.

Bayley, P.B. (1991). The flood pulse advantage and the restoration of river-floodplain system, Regulated Rivers: Research and Management, 6, 75-86.

Brandt, S.A. (2000). Classification of geomorphological effects downstream of dams. Catena, (40), 375-401.

Bunn S.E., Arthington A. (2002). Basic principles and ecological consequences of altered flow regimes for acuatic biodiversity, Enviromental Management 30(4), 492-507.

Cabrero Rodríguez, A. y Magdaleno Mas, F. (2015). Buenas prácticas para el mantenimiento y conservación de cauces. Revista Digital Del Cedex, (177), 51. Recuperado de http://ingenieriacivil.cedex. es/index.php/ingenieria-civil/article/view/517

Conesa García, C., y Pérez Cutillas, P. (2014). Alteraciones geomorfológicas recientes en los sistemas fluviales mediterráneos de la Península Ibérica: Síntomas y problemas de incisión en los cauces. Revista de Geografía Norte Grande, 59, 25-44. Confederación Hidrográfica del Guadalquivir (2002). Fichas ténicas y dípticos de presas.

Demarcación Hidrográfica del Guadalquivir. Planes Hidrológicos de la Demarcación del Guadalquivir. Recuperado de https://www.chguadalquivir.es/ demarcacion-hidrografica-guadalquivir

Frutos, L. M., Ollero, A., y Sánchez Fabre, M. (2004). Caracterización del Ebro y su cuenca y variaciones en su comportamiento hidrológico. En Gil Oncina, A. (Ed.), Alteración de los regimenes fluviales peninsulares, pp. 223-280, Fundación Caja Murcia, Alicante.

García Martínez, B. (2016). Cambios hidromorfológicos en el Guadalquivir y sus principales afluentes: Guadalbarcar, Rivera de Huézar y Rivera de Huelva (Sector, Palama del Río-Sevilla), Tesis Doctoral, Universidad de Sevilla.

García Martínez, B. y Baena Escudero, R. (2001). Los embalses de regulación como inductores de cambios hidrológicos y morfológicos en los ríos de An- dalucía occidental, En Actas del XVII Congreso de Geógrafos Españoles, (pp.163-165), Oviedo.

García Martínez, B. y Baena Escudero, R. (2002). Nuevas Aportaciones a la Caracterización Hidrogeomorfológica de los Ríos de Sierra Morena Occidental: la Cuenca del Río Rivera de Huéznar (Sevilla). En Estudios Recientes (2000-2002) en Geomorfología. Patrimonio, Montaña y Dinámica Territorial, (pp 503-512), Valladolid. SEG-Departamento de Geografía Universidad de Valladolid.

García Martínez, B. y Baena Escudero, R (2007). Comportamiento Hidrogeomorfológico del Río Rivera de Huéznar (Sierra Morena, España), Revista de Geografía, 9(11) 36- 46.

García Martínez, B., Baena Escudero, R. y Posada Simeón, J.C. (2009). La acción antrópica como inductora de cambios en el patrón fluvial del río Rivera de Huelva (sector Guillena-Sevilla), En Romero, M.A., Belmonte, F. Alonso, F. López Bermúdez, F., (Eds.), Avances en estudio sobre Desertificación (pp 472-476), Murcia, Universidad de Murcia.

García Martínez, B. y Ruíz Miranda, S. (2017). Cambios en el comportamiento hidrológico reciente inducido por la acción del hombre: el caso del río Bembézar (Cuenca del Guadalquivir, España). En Allende Álvarez et al. (Eds.), Naturaleza, Territorio y Ciudad en un mundo global (pp 145-153), Madrid, Asociación de Geógrafos Españoles

Gil Olcina, A. (2006). Regímenes natural y artificial del río Júcar, Investigaciones Geográficas, 40, 5-34.

Herrera Grau, T. (2014). De las "limpiezas de ríos" a la "conservación y mantenimiento de ríos": prevención de riesgos, conservación y empleo pueden darse la mano. Recuperado de https://fnca.eu/images/ documentos/DOCUMENTOS/Tony_Herrera.pdf

Lorenzo-Lacruz, J., Vicente-Serrano, S. M., López-Moreno, J. I., Morán-Tejeda, E., y Zabalza, J. (2012). Recent trends in Iberian streamflows (19452005). Journal of Hydrology, 414, 463-475.

Martínez-Castroviejo, R., Gómez-Villar, A. y García-Ruíz, J.M. (1991). Ajustes fluviales derivados de cambios de usos del suelo en el Pirineo Aragonés, Cuaternario y Geomorfología, 5, 91-105.

Martínez Santa-María, C. y Fernández Yuste, J.A. (2006). El régimen natural de caudales: una diversidad imprescindible, una diversidad predecible. Investigación agraria. Sistemas y recursos forestales, 15 (1), 153-165. 
Masachs Alavedra, V. (1948). Los factores determinantes de la distribución espacial de los tipos de régimen de los ríos de la Península Ibérica, Estudios Geográficos, 28, 475-492.

Ministerio de Medio Ambiente y Medio Rural y Marino (2011): Informe hidrológico 2010-2011. Cuenca Hidrográfica del Guadalquivir. Dirección Técnica, Servicio de Explotación.

Ministerio para la Transición Ecológica (2019). Protocolo de caracterización Hidromorfológica de masas de agua de la categoría ríos. Código M-RHMF-2019.

Ollero, A. (2008). Alteraciones geomorfológicas de los ríos en Europa y principios para la restauración de su dinámica. Ríos y sostenibilidad. Semana temática "Agua para la vida». Tribuna del Agua, Exposición Internacional de Zaragoza.

Ollero, A. (2013). ¿Por qué no hay que limpiar los ríos? Centro Ibérico de Restauración Fluvial. CIREF. Recuperado de http://www.cirefluvial.com/noticias_ver.php?id=225

Ollero, A., Sánchez, M., Marín, J. M., Fernández, D., Ballarín, D., Mora, D., \& Zúñiga, M. (2004). Caracterización hidromorfológica del río Gállego. En: JL. Peña, L.A. Longares y M. Sánchez (Eds.), Geografia Física de Aragón. Aspectos generales y temáticos, Universidad de Zaragoza e Institución Fernando el Católico. Zaragoza, 2004.

Ollero, A., Ballarín Ferrer, D., Díaz Bea, E., Mora Mur, D., Sánchez Fabre, M., Acín, V., Echevarría Arnedo, M.T., Granado García, D., Ibisate González de Matauco, A., Sánchez Gil, L.y Sánchez Gil, N. (2008). IHG: un índice para la valoración hidrogeomorfológica de sistemas fluviales. Limnetica, 27(1), 171-188.

Park, C.C. (1977). Man-induced changes in stream channel capacity. In Gregory, K. J (Ed.), River channel changes, 121-144, Wiley.

Petts, G.E. (1984). Sedimentation within a regulated river, Earth Surface Processes and Landforms 9 (2), 125-134.

Remenieras, G. (1971). Tratado de hidrología aplicada. Editores Técnicas Asociadas, S.A. Barcelona.

Rinaldi, M., Surian, N., Comiti, F., Bussettini, M. (2013). A method for the assessment and analysis of the hydromorphological condition of Italian streams: the Morphological Quality Index (MQI). Geomorphology 180-181, 96-108
Rinaldi, M., Belletti, B., Bussettini, M., Comiti, F., Golfieri, B., Lastoria, B., Marchese, E., Nardi, L., Surian, N. (2017). New tools for the hydromorphological assessment and monitoring of European streams. Journal of Environmental Management, 202, 363-378

Rinaldi, M., Baena Escudero, R., Nardi, L., García Martínez, B., Guerrero Amador, I. (2018). Hydromorphological analysis of the Guadalquivir fluvial system (Southern Spain). [Technical report]. Universidad de Sevilla.

Rinaldi, M., Baena Escudero, R., Nardi, L., Guerrero Amador, I.C, García Martínez, B. (2020). An assessment of the hydromorphological conditions of the middle and lower Guadalquivir River (southern Spain). Physical Geography. 2020. 41, (3), 254-271.

Schumm, S.A. (1977). The fluvial System. A Wiley-Interscience Publication.

López Bermúdez, F. (2004). El Segura, la alteración del régimen de un rıo mediterráneo y su tendencia al debilitamiento. En Gil Oncina, A. (Ed.), Alteración de los regimenes fluviales peninsulares, pp. 389411, Fundación Caja Murcia, Alicante.

Stanley, J.M. (1972). Retrogression on the low Colorado River after 1935. Trans. Am. Soc. Engrs, 116, 943-957.

Stepánek, P. (2005). AnClim-software for time series analysis. Dept. of Geography, Fac. of Natural Sciences, Masaryk University, Brno.

Talavera, J. M., y Sánchez, R. N. (2019). Aplicación del Índice Hidrogeomorfológico (IHG) en la cuenca del Segura: Embalse de la Fuensanta-Llano de la Vida (Desembocadura del río Taibilla). GeoGraphos: Revista Digital para Estudiantes de Geografía y Ciencias Sociales, 10(120), 238-268.

Thornes, J.B. (1977). Hydraulic geometry and channel change. In Gregory, K. J (Ed.): River Channel Changes, Wiley, 91-100 Chichester.

Vanney, J.R. (1970). L'Hydrologie du Bas Guadalquivir. Instituto de Geografía aplicada del Patronato "Alonso de Herrera". C.S.I.C.

Vericat, D. y Batalla, J.R. (2004). Efectos de las presas en la dinámica fluvial del curso bajo del río Ebro, Rev. C. \& G., 18 (1-2), 37-50.

Williams, G. P., \& Wolman, M. G. (1984). Downstream effects of dams on alluvial rivers. Washington, DC: US Government Printing Office. 